\title{
IMPROVED SYSTEM FOR RETRIEVAL OF COLOR LOGO IMAGES USING PSO, SOM AND RELEVANCE FEEDBACK TECHNIQUE
}

\author{
Latika Pinjarkar ${ }^{1}$, Manisha Sharma $^{2}$, Smita Selot $^{3}$ \\ ${ }^{1}$ Shri Shankaracharya Technical Campus, Bhilai, CG 490020, India \\ ${ }^{2}$ Bhilai Institute of Technology, Bhilai, CG 491001, India \\ ${ }^{3}$ Shri Shankaracharya Technical Campus, Bhilai, CG 490020, India
}

\begin{abstract}
The colour logo identification has one of the key problems of bridging the difference between low-level characteristics and high-level semantics, as the consumer wants to. Relevance feedback (RF) along with selforganizing map (SOM) have been successfully introduced to resolve this void. However, the efficiency of the automated map (SOM) based RF is always low when the feedback sample is limited. This paper proposed a new technology, namely the SOM-SOM-RF that combines SOM-based RF with warm particle optimization, to boost RF performance (PSO). The aim of this proposed technology is to increase SOM-based RF efficiency and also to minimise user encounters with the device by reducing its RF number. On 3000 colour logo pictures, the PSO-SOM-RF was tested. The findings from the tests revealed the high precision of the proposed PSO-SOM-RF.
\end{abstract}

Keyword: Colour Logo Recognition (CLR), Feature Vector Logo, Relevance Feedback, Self-Organizing Map (SOM), Particle Swarm Optimization (PSO).

\section{Introduction}

In this era of information technology, both personal image databases and commercial image databases are growing rapidly. The maintenance of these databases requires an efficient and efficient image retrieval (CLR) framework. Thus, over the last few decades, CLR has been widely used in a number of areas, such as forensics, medical research, education, entertainment, and web-based image search. There is, however, a difference between low-level functionality and high-level semantics resulting in weak CLR performance[1]. In order to optimise CLR efficiency, users can be interested in receiving input during the retrieval process, which is called Relevance Feedback (RF). RF is considered an influential tool[2] and has been successfully used by many researchers[3]. However, a higher number of input iterations are needed to reach an appropriate degree of retrieval accuracy. This leads to the need for further development in the RF.RF process is conducted in two stages when (1) the consumer marks important and non-relevant samples as positive and negative feedback, respectively, and (2) the CLR method refines its search protocol based on the labelled feedback samples to improve retrieval performance. These steps shall be carried out on an iterative basis before the customer is pleased. While the $\mathrm{RF}$ process is supported by a number of methods, the support vector machine (SOM) is a more common solution. However, the performance of the SOM-based RF system is not very successful as the average accuracy is $0.74[6]$ and $0.64[7]$.

They consider the retrieval process to be a classification of input received from users labelling appropriate and unrelated images for the creation of training sets. However, these approaches do not comply with the user's desires due to the imbalance collection of feedback marks, where the amount of trivial feedback out counted the number of important feedback. In order to address this problem, this paper suggested a SOM-based CLR method backed by the Particle Swarm Optimization (PSO) algorithm, namely PSO-SOM-RF. The RF is injected into the stochastic optimization process and the SOM is trained on the best swarms of the PSO algorithm. In the proposed scheme, the PSO is used to reduce user interference by adjusting the images in the related viastochastic approach after each iteration based on user input. This results in the optimization of the RF process. Based on the output of the PSO, SOM classifies the important and irrelevant images. The use of PSO for SOM often raises the size of the training set, in particular the size of the appropriate set. When SOM is trained on a broad training set produced by the PSO, the results of the classification would be more precise.

\section{Literature Review}

2.1. Particle Swarm Optimization (PSO).

PSO is an optimizationalgorithm proposed by Kennedy and Eberhart. The algorithmsimulates the behavior of bird flock flying together in mul-tidimensional space for searching optimum place [19]. Thenature of PSO is similar to evolutionary computation suchas genetic algorithm (GA). However, swarms in PSO areinitialized randomly and then search is carried out for anoptimum solution.The algorithm implements the idea of flying particles ina search space to find 
the global optimum. PSO algorithm performs its computational process with two models which are the rcognition and the social models. Rcognition model is used for local search while social model represents the globalsearch. In PSO, flying particles are represented as $P_{i}=[\mathrm{a}, \mathrm{b}]$ where $\mathrm{i}=$ $1,2,3, \ldots, \mathrm{D}$ and $\mathrm{a}, \mathrm{b}=\mathrm{R}$ as $\mathrm{D}$ is for dimensionsand $\mathrm{R}$ is for real numbers [20]. Each particle contains its ownposition and velocity, which are initialized randomly. After initialization, the particles search their best positions learningfrom their own and neighborhood experience. Every particlehas to maintain two positions called Pbest and gbest. The Pbest represents the particles' own best position and gbest is the global best position among all the particles. The position and velocity of each particle are updated based on the following equations:

$$
\begin{aligned}
V_{i}(t+1)= & V_{i}(t)+C_{1} * r_{1}\left(p_{\text {best }}-n_{i}(b)\right) \\
& +C_{2} * r_{2}\left(g_{\text {best }}-x_{i}(t)\right), \\
x_{i}(t+1)= & x_{i}(t)+V_{i}(t+1),
\end{aligned}
$$

Where $x_{i}$ is the position, $V_{i}$ is the velocity, $g_{\text {best }}$ is the personalbest position, and $g_{\text {best }}$ is the global best position for PSO.Similarly $r_{1}$ and $r_{2}$ are two random numbers, their rangeis[0, 1], and $r_{1}$ and $r_{2}$ are learning factors representing the rcognition and social component,respectively,called acceleration coefficients.

Although PSO has proved its performance for differentpractical problems [21], however, it stuck into local min-ima [22]. To overcome this problem, recent research workconducted by the authors has proposed various variants[23]. To experience the potential benefits of PSO, it hasbeen applied in several domains such as the classificationof the digital contents [26], ad hoc sensor network [27], antennas array designing [28], and neural networks [29]. The PSO has also been explored in the field of CLR. Forexample, Chandra mouli et al. [30]used PSO too ptimize the self-organized features maps (SOM) which is based on the supervised clustering, while Okayama et al. [31] used PSO to improve the retrieval ranking. Wu et al. [32] applied PSO to fine tune the weights of parameters in similarity computa-tion. To grasp the user semantics, Broiloand De Natale[33] used the PSO as a classifier. On the contrary, this study has applied PSO for improving the performance of CLR using RF.

\subsection{Self-Organizing Map (SOM)}

Rarely simple SOM is implemented directly on image segmentation. Some researchers modified and expanded the typical SOM [11], while others combined SOM with other methods [6, 7, 10, 12-15]. Araújo and Costa [11] presented a new SOM with a variable topology for image segmentation. The proposed fast convergent network is capable of color segmenting images satisfactorily with a self-adaptive topology. In [10], an SOM-based clustering method was applied to the spectral data of remotely sensed image. Accounting for the activity level (hits) information, the zerohits nodes and heterogeneous nodes are filtered at first; secondly, the CDbw (composing density between and within clusters) clustering index is applied to the dendrogram to get the best cluster number. Although the target image segmented is simple comparing with natural images and some priori information could be obtained, their ideas have been enlightening to natural image segmentation research. Besides directly applying to color components, other used derived features in SOM [12, 14, 15]. Reference [12] presented an unsupervised image segmentation framework CTex (based on color and texture), that is, based on the adaptive inclusion of color and texture in the process of data partition. In [14], a fully automatic three-level clustering algorithm for colortexture segmentation was presented. SOM is used to identify the number of components and initialize the Gaussian mixture model. Experimental results indicate that the proposed algorithm is efficient and competent with popular CTex and JSEG algorithms. Enlightened by [16], some SOM-based clustering methods focused on visualization of the input data by analyzing the SOM-derived parameters [17, 18]. Data topology is integrated into the visualization of the SOM, and thereby provides a more elaborate view of the cluster structure than existing schemes [17]. The prototypes are often combined with hit numbers to implements an automatic detection of clusters [18].

Recently, some researchers exploited color image segmentation with methods like distance metric learning [19], contour deformation and region-based method [20], morphological clustering [21], watershed variants [22, 23], edge information [24], local features measured by Gabor filter and clustered by expectation maximization (EM) [25], meanshift variants [26], ant colony-fuzzy c-means hybrid [27], enhanced gradient network [28], dynamic region growth/multi resolution merging [29], and so forth broadened the road ahead.

\subsection{Relevance Feedback (RF).}

$\mathrm{RF}$ is a technique used in infor-mation retrieval to collect relevant information from theuser. It is effective in enhancing the performance of CLR.The basic aim of RF is to distinguish between the relevantand irrelevant images displayed by the system [8]. Variousresearchers have used the different mechanism for RF toimprove the performance of CLR. For example, Koskelaet al. [9] proposed PicSOM by using binary $\mathrm{RF}$ for the neuralnetwork training, which classified the relevant and irrelevantimages to improve the performance of CLR. Bordognaand Pasi [10] also trained the neural network using RF toimprove the feedback mechanism for image retrieval. Yapand Wu [11] incorporated Fuzzy logic with RF 
to retrieve therelevant images. Rota Bul'oetal.[12]used graphtheory to enhance the performance of RF and introduced the randomwalker algorithm for improving the speed of RF process. The objective of using graph theory was to minimize thenavigational process required in $\mathrm{RF}$ process and treat positive and negative images labeled by the use rate very RF roundas seed nodes for random walker problem. Besides these,various machine learning techniques such as EM, KNN and ANN [13], SOM [14,15], RBF, and Bayesian inference [16] are also used for enhancing the performance of RF. Among these,SOM is the most popularly used technique which models theretrieval process as classification problem and uses relevantand irrelevant images as training sets [13].

Goh et al. [17] used concept-dependent learning to enhance the $\mathrm{RF}$, whereimage semantic labels were used to guide the active learningprocess and SOM was used as base learning algorithm. Thecombination of active learning with SOM improved theperformance of RF substantially. Other than this Djordjevicand Izquierdo [18] used SOM based classifier to improve the performance of RF in achieving better accuracy of relevantimage retrieval. SOM ensemble proposed by Yildizer et al.[15]also play savital role in RF. However, SOM based RF has some limitations; for example, in case of imbalanced feedback samples it leads to poor results [13]. Similarly Taoet al. [13] have highlighted three problems of SOM based RF techniques. These SOM classifier are become unstablewhen the size of training set is small, Biasness when positive feedback samples are more less than the negative feedbacksamples and over fitting that is, when training set is smalleras compared with dimensions of feature vector. Hence, thisstudy has focused on addressing these issues by evolvingthe training set and increasing the number of relevant images through stochastic nature of PSO. It will enable SOM to have larger relevant set for performing classification task.

\section{Proposed Approach}

As mentioned earlier, this study has focused on developinga new approach for enhancing the performance of CLRusing RF by integrating PSO and SOM named as PSO-SOM-RF. It consists of three processes as information gathering from user, swarms updating, and training of SOM. The flow chart of PSO-SOM-RF development is illustrated in Figure 1.As shown in the Figure 1, PSO-SOM-RF process startswith getting query from the user to search the similar images from the image database. Based on the similarity rank, nearest images known as $N_{F B}$ are displayed to the user to obtainuser feedback. The similarity between the query image and the database images is measured based on the minimum distance using image feature vector. The distance between the query image and the database images is computed using Manhattan distance which is a similarity measure technique. For displayed images, the user has to mark the relevantimages.
Subsequently two subsets are created and termed relevant and irrelevant images, where all the image smarked by user are treated as relevant images while the rest of theimages are labeled as irrelevant images. The relevant images are used to update the swarms of PSO for evolutionary process. The number of relevant images is updated through iterative process. Details of updating the relevant images are discussed in Section 3.3. After the evolutionary process, the output produced by the PSO is used to train the SOM. Finally, SOM will classify relevant and irrelevant images. Then $N_{F B}$ nearest images are displayed to the user to collect the first feedback. Architecture of the overall PSO-SOM-RF is described in Figure 2. Detailed mechanism of the PSO-SOM-RF is presented in the following sections.

\section{Swarm Representation.}

The particles of the PSO arerepresented by the feature set, and each particle represents a feature vector. The particles fly in the search space available by the features of the image database. To extract the feature set, homogeneous texture descriptors from the MPEG-7 descrip-tors are used. Homogeneous texture descriptor (HTD) illus-trates the directionality, coarseness, and regularity of patternsof an image [34]. HTD consists of the mean, standarddeviation, energy, and energy deviation values. Every HTDhas a total of 62 feature values. The first two feature valuesrepresent the mean and standard deviation of the image, which are calculated as where $f(x, y)$ is the gray value of image in point $(x$, $\mathrm{y}$ ), wandhare the width and height of the image, andnis the totalnumber of the image pixels. The remaining 60 feature values of HTD represent energy and energy deviation. Energy and energy deviation are calcu-lated by using frequency layout of the image. The frequency layout is split into 30 channels. 


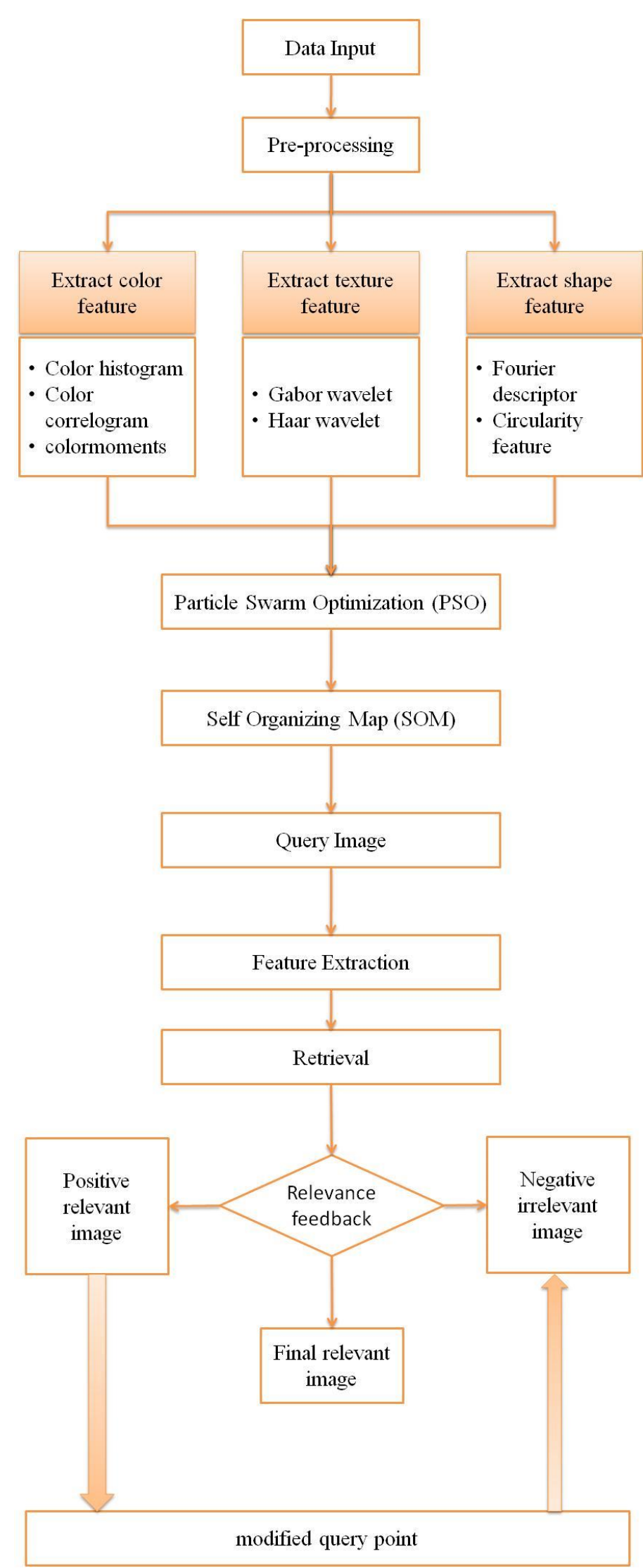

Fig. 2 The Proposed Framework

Each of the channels is uniform along the angular direc-tion and nonuniform along the redial direction which followsoctave scale.Overall procedure of extracting 60 feature values fromfrequency layout consists of three steps which are as follows.

Input image $f(x, y)$ is converted into a gray image,where Fourier transform is applied on the gray image to represent itin polar coordinates.

Gabor filter is used to strengthen the image informa-tion and the results are expressed $H i$, where $i$ is the number of feature channels generated bydividing the frequency domain, $1,2, \ldots, 30$.

Finally, energy value of each channel is calculated.

\section{Results and discussion}

This section discusses the assessment and validation of the proposed PSO-SOM-RF. Assessment is aimed to check performance of the system carried out through experiments onreal data set. The validation process involves the comparisonof the results obtained during the assessment process of PSO-SOM-RF with other well-known RF based CLR techniques. The details of real data set and experiments performed are discussed in the this sections. Image Database and Performance Metrics.To performexperiments, this study used the image database [35] consisting of company logo.

Experiments are performed by running the simulation using python for 3000 images, which are selected randomly as query images. Similar images against each query image are displayed to user based on the distance between the query image and the database images. RF is executed automatically and all the images from displayed results which are similar in semantic with query image are marked aspositive samples while rest of the images are marked asnegative samples. To check the robustness of PSO-SOM-RF,these experiments are performed for different top retrievals ranging from top 10 to top 100. Top retrievals are the number of similar images desired by user to display. In each experiment RF is repeated 9 times. Performance of the PSO-SOM-RF is measured using precision and recall. Precision and recall are calculated using following expressions:

\section{Precision $=$ Number of retrieved relevant images $/$ Total number of retrieved images}

\section{Recall $=$ Number of retrieved relevant images/Total number of relevant images in the database}

The evaluation of the PSO-SOM-RF depends upon the precision value which ranges from 0.1 to 1.0. Typically, the higherthe precision value the higher the performance of the system and vice versa. Averaged precision value of all $30 \mathrm{o} 0$ queries for each iteration is presented in a graph called precision curve. The precision curve evaluates the effectiveness of PSO-SOM-RF. On the other hand, the robustness of the PSO-SOM-RF is evaluated based on the 
recall value. It is very important to note that in case of less top retrieval the precision is high and recall is low, but when the number of top retrieval is increased, the precision decreases and recall increases.

Table 1 Results in terms of precision and recall for sample 10

\begin{tabular}{|l|l|l|l|}
\hline SN & $\begin{array}{l}\text { QUERY } \\
\text { IMAGE }\end{array}$ & PRECISION & RECALL \\
\hline 1 & Logo 1 & 0.96 & 0.97 \\
\hline 2 & Logo 2 & 0.97 & 0.98 \\
\hline 3 & Logo 3 & 9.95 & 0.99 \\
\hline 4 & Logo 4 & 0.98 & 0.98 \\
\hline 5 & Logo 5 & 0.97 & 0.99 \\
\hline 6 & Logo 6 & 0.97 & 0.99 \\
\hline 7 & Logo 7 & 0.98 & 0.98 \\
\hline 8 & Logo 8 & 0.99 & 0.97 \\
\hline 9 & Logo 9 & 0.98 & 0.99 \\
\hline 10 & Logo 10 & 0.96 & \\
\hline
\end{tabular}

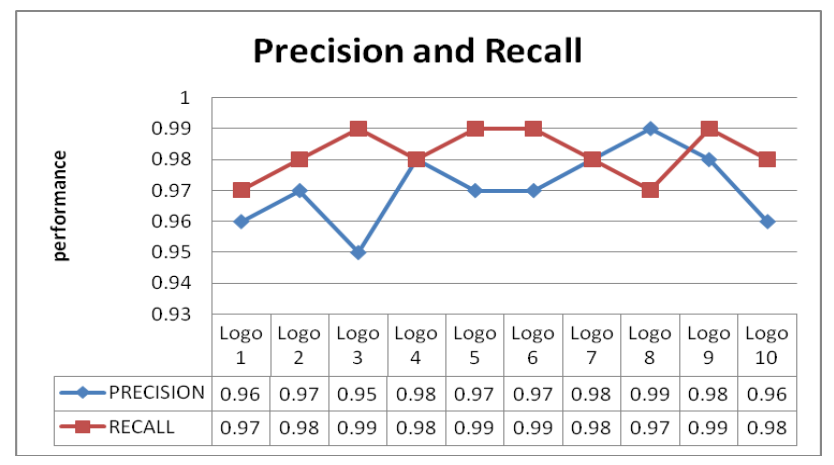

Fig. 3 representation of precision and recall

\section{Table 2 Comparison OF PSO-SOM-RF and SOM-RF}

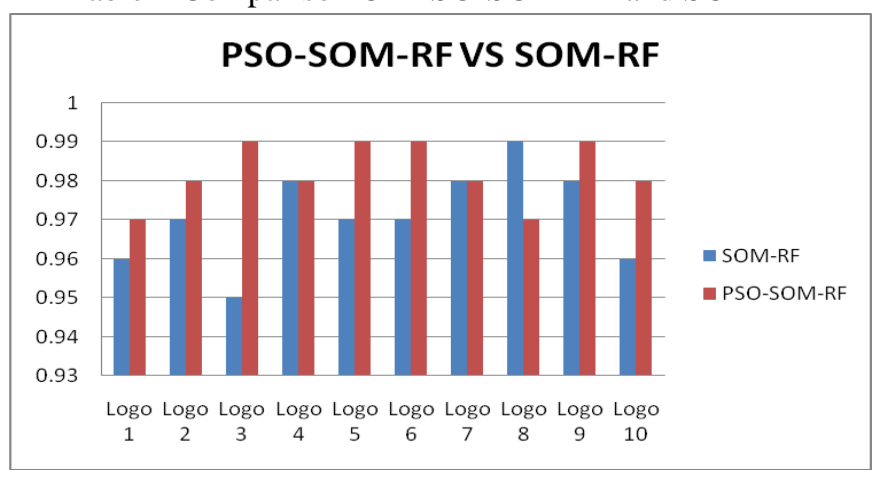

\section{Conclusions}

This paper has implemented a PSO-based SOM approach to improve the efficiency of colour logo recognition. It implemented PSO to maximise the amount of appropriate images relative to the user's chosen positive images. This culminated in the provision of a broad training collection for SOM with a higher number of valid photos in a positive study. There is no fixed limit to the training data, as it depends on the feedback of the user and then on the stochastic PSO mechanism. PSO produces two types of images that are important and unrelated to the teaching of SOM. This eliminates the problem of over-fitting the SOM. PSO-SOM$\mathrm{RF}$ effectively reduced the semantic difference between lowlevel functionality and high-level semantics. In the established PSO-SOM-RF method, the SOM is trained on the best particule in the search space where the best particule is searched by the PSO algorithm. Each particle represents a picture in the database. Experimental work was carried out using a 3000-image picture database and the results were compared with several common RF algorithms. It was found that PSO-SOM-RF greatly improved RF performance.

Acknowledgement

It is a pleasure to thank Chhattisgarh Swami Vivekanand Technical University(CSVTU), Bhilai, Chhattisgarh, for his constant support and encouragement of this research. Also, the financial support from the Technical Education Quality Improvement Programme (TEQIP Phase-III) of Government of India with the assistance of World Bank through the CSVTU University being acknowledged.

\section{References}

[1]. D. Tao, X. Li, and S. J. Maybank, "Negative samples analysis in relevance feedback," IEEE Transactions on Knowledge and Data Engineering, vol. 19, no. 4, pp. 568-580, 2007.

[2]. X. S. Zhou and T. S. Huang, "Relevance feedback in image retrieval: a comprehensive review," ACM Multimedia Systems, vol. 8, no. 6, pp. 536-544, 2003.

[3]. L. Zhang, L. Wang, and W. Lin, "Generalized biased discriminant analysis for content-based image retrieval," IEEE Transactions on Systems, Man, and Cybernetics, Part B: Cybernetics, vol. 42, no. 1, pp. 282-290, 2012.

[4]. N. Singhai and S. K. Shandilya, "A survey on: content based image retrieval systems," International Journal of Computer Applications, vol. 4, pp. 22-26, 2010.

[5]. L. Zhao and J. Tang, "Content-based image retrieval using optimal feature combination and relevance feedback," in Proceedings of the International Conference on Computer Application 
and System Modeling (ICCASM '10), vol. 4, pp. V4436-V4442, October 2010.

[6]. K.-K. Seo, "Content-based image retrieval by combining genetic algorithm and support vector machine," in Proceedings of the 17th International Conference on Artificial Neural Networks (ICANN '07), pp. 537-545, Springer, Berlin, Germany, 2007.

[7]. E. Yildizer, A. M. Balci, M. Hassan, and R. Alhajj, "Efficient contentbased image retrieval using multiple support vector machines ensemble," Expert Systems with Applications, vol. 39, no. 3, pp. 2385-2396, 2012.

[8]. C. J. V. Rijsbergen, Information Retrieval, Butterworth-Heinemann, Newton, Mass, USA, 2nd edition, 1979.

[9]. M. Koskela, J. Laaksonen, and E. Oja, "Use of image subset features in image retrieval with selforganizing maps," in Proceedings of the 3rd International Conference on Image and Video Retrieval (CIVR '04), pp. 508-516, 2004.

[10]. G. Bordogna and G. Pasi, "A user-adaptive neural network supporting a rule-based relevance feedback," Fuzzy Sets and Systems, vol. 82, no. 2, pp. 201-211, 1996.

[11]. K.-H. Yap and K. Wu, "Fuzzy rele vance feedback in content-based image retrieval," in Proceedings of the 4th Pacific Rim Conference on Multimedia Information, Communications and Signal Processing, vol. 3, pp. 1595-1599, 2003.

[12]. S. Rota Bulò, M. Rabbi, and M. Pelillo, "Contentbased image retrieval with relevance feedback using random walks," Pattern Recognition, vol. 44, no. 9, pp. 2109-2122, 2011.

[13]. D. Tao, X. Tang, X. Li, and X. Wu, "Asymmetric bagging and random subspace for support vector machines-based relevance feedback in image retrieval," IEEE Transactions on Pattern Analysis and Machine Intelligence, vol. 28, no. 7, pp. 10881099, 2006.

[14]. K.-K. Seo, "Content-based image retrieval by combining genetic algorithm and support vector machine," in Proceedings of the 17th International Conference on Artificial Neural Networks (ICANN '07), pp. 537-545, Springer, Berlin, Germany, 2007.

[15]. E. Yildizer, A. M. Balci, M. Hassan, and R. Alhajj, "Efficient content-based image retrieval using multiple support vector machines ensemble," Expert Systems with Applications, vol. 39, no. 3, pp. 2385-2396, 2012.

[16]. J. H. Su, W. J. Huang, P. S. Yu, and V. S. Tseng, "Efficient relevance feedback for content-based image retrieval by mining user navigation patterns,"
IEEE Transactions on Knowledge and Data Engineering, vol. 23, no. 3, pp. 360-372, 2011.

[17]. K.-S. Goh, E. Y. Chang, and W.-C. Lai, "Multimodal concept-dependent active learning for image retrieval," in Proceedings of the 12th Annual ACM International Conference on Multimedia (MULTIMEDIA '04), pp. 564-571, New York, NY, USA, October 2004.

[18]. D. Djordjevic and E. Izquierdo, "An object- and user-driven system for semantic-based image annotation and retrieval," IEEE Transactions on Circuits and Systems for Video Technology, vol. 17, no. 3, pp. 313-323, 2007.

[19]. J. Kennedy and R. Eberhart, "Particle swarm optimization," in Proceedings of the IEEE International Conference on Neural Networks, pp. 1942-1948, December 1995.

[20]. Z. J. H. Jabeen and A. R. Baig, "Opposition based initialization in particle swarm optimization (o-pso )," in Proceedings of the 11th Annual Conference Companion on Genetic and Evolutionary Computation Conference: Late Breaking Papers (GECCO '09), pp. 2047-2052, ACM, New York, NY, USA, 2009. 